Introduction

The integration of concealable stigmatized identities (CSI) is an active process that requires conscious effort and planning. CSIs are defined as "identities that can
be hidden from others and that are socially devalued be hidden from others and that are socially devalued and negatively stereotyped" (Quinn \& Earnshow, 2013) Non-governmental organizations (NGOs) must dired resources and funding to these efforts in order to achieve optimal outcomes. Many NGOs work passively to integrate CSIs; however, this can often confuse those Culture plays a critical role in understanding this unique construct (lkizer, Ramírez-Esparza, \& Quinn, 2018); and those living with CSIs indicate different needs across cultures, resulting in NGO programing needing to take this diversity into account.

NGOs may be serving diverse populations with multiple concealable stigmatized identities. For example, NGOs may work directly with individuals who have a history of mental illness or incarceration, and these identities impact how members benefit from and perceive NGO programming and support. NGOs must be intentional in taking these identities into consideration to be most effective in their program outcomes.

A one size fits all approach for integrating CSIs should be avoided given the potential for immense cultural diversity.
PovSolve as a Case Study

PovSolve has worked across the world with various religious, sexual, and cultural identities. A prominent example of integration efforts of these identities specific to India is our homeless feeding program for those living in poverty located in a religious neighborhood. This often results in those identifying as LGBTQ or gay being unable to feel comfortab and inclusive in taking advantage of the program offerings.

Being an NGO that has worked across diverse countries and cultures, this NGO has had the unique opportunity to interact, both drectly and harecly, wint .

The following strategies have proven effective for PovSolve: Multi-modal communication approaches to reach those with CSIs who may not be forthcoming in directly and openly stating their needs

Creating safe spaces, both physically and virtually, across the program for those with CSI's to indirectly share their perspectives and needs without being pressured or in the spotlight

Stigma management training with program staff and volunteers to create awareness of this critical topic

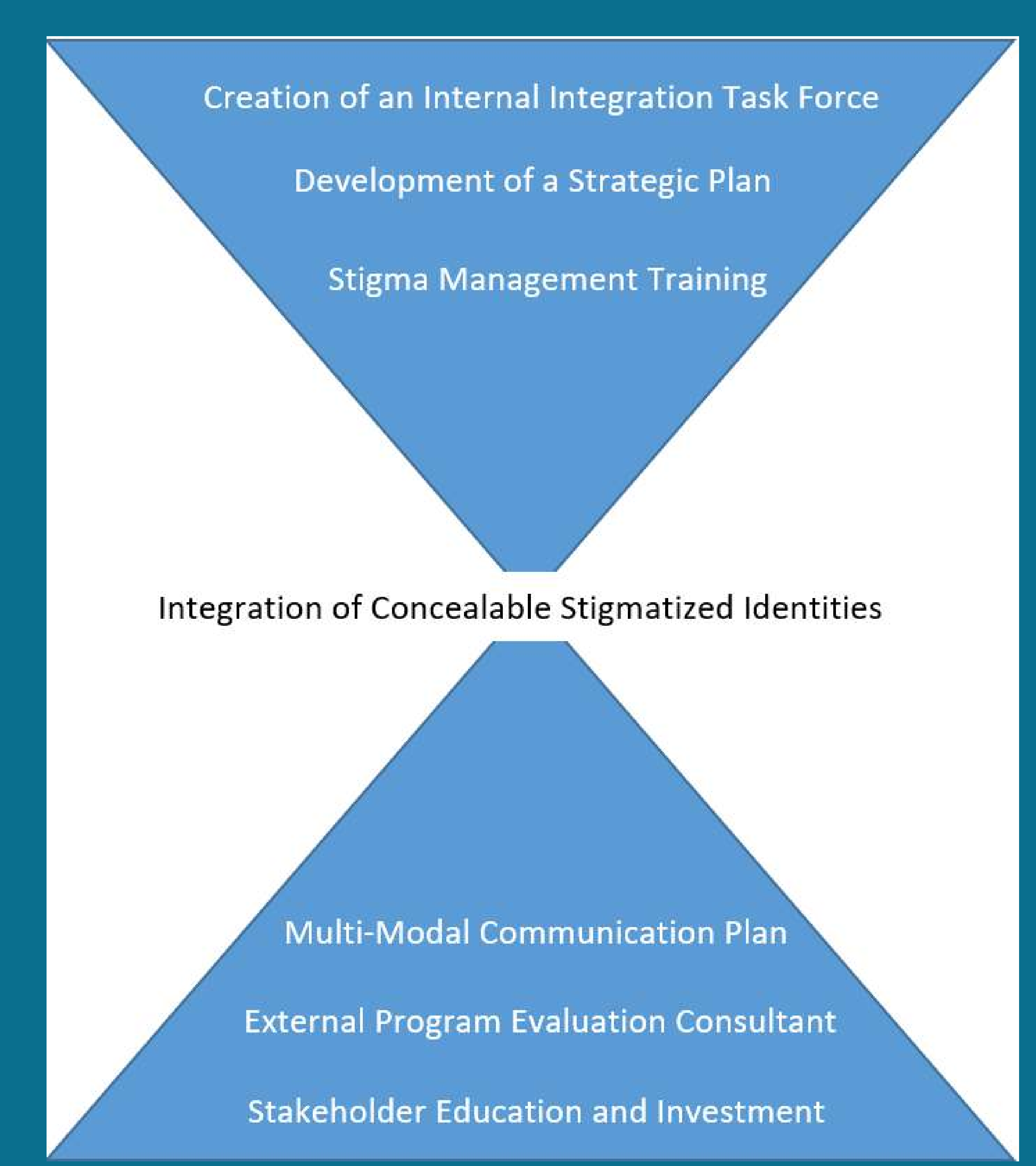

Potential Strategies for Integration

1) Identify and understand which concealable stigmatized Identities exist in an NGO area of progranming (Quinn, 2006).

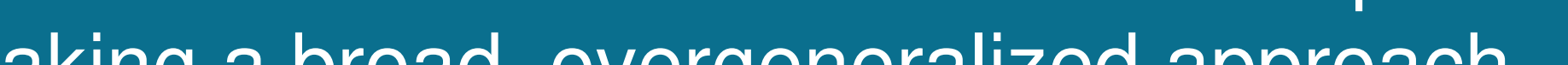

2) Determine the specific needs of those communities using collaborative methods such as community-based participatory research (Holkup, Tripp-Reimer, Salois, \& Weinert, 2004

3) Create an internal diversity or inclusion task force within the NGO that is specifically focused on integration programming for those with concealable stigmatized identities.

4) Proactively implement measures to manage stigma within the NGO and throughout the organizational programming because NGO workers can unintentionally stigmatize those who they serve (Nambiar, 2011).

5) Respect confidentiality of those who share their concealable dentities with friends and family, but keep the information private from professional colleagues. This includes avoiding soliciting this
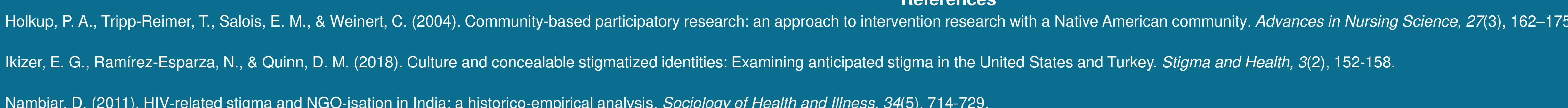

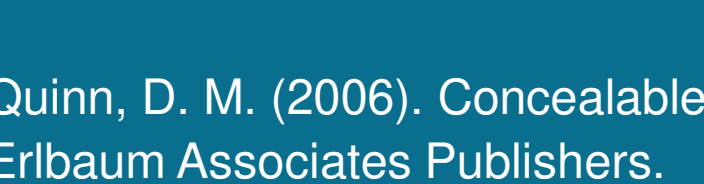

\section{May 2019}

NGO creates an internal task force to implement integratio strategy. Task force should include key stakeholders.

7 There will be a lead facilitator

for meetings \& conduct

decision-making group work within the task force.

$M$

E

L

I

$\mathbf{N}$

$E$

\section{July 2019}

Hire an external consultan

to lead the research and

strategy efforts. The use of

a third party can reduce

the stigma around the

topic within the NGO

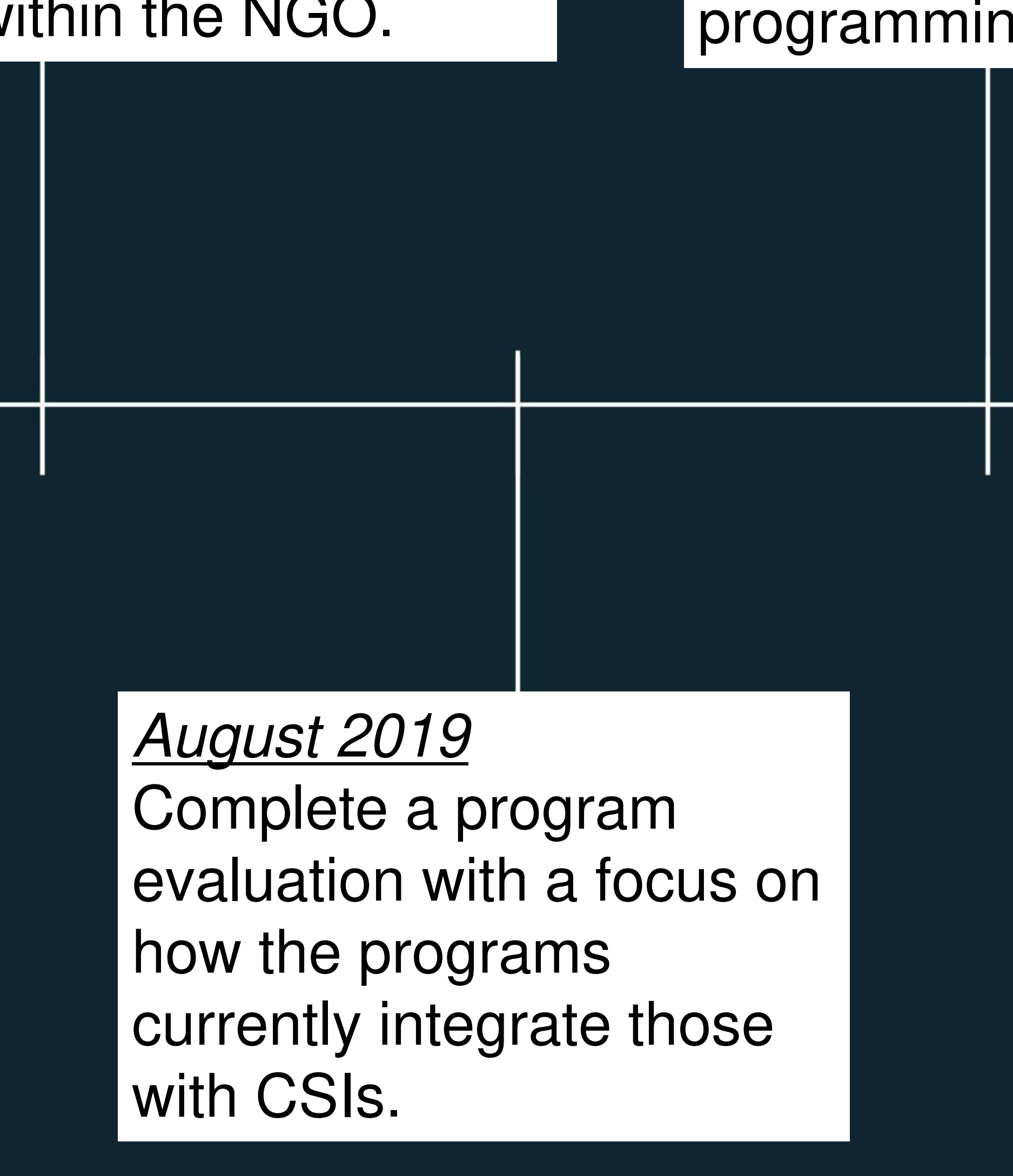

September 2019

During the program

evaluation, use

qualitative methods to

earn more about the

Is in the NGO

ramming.

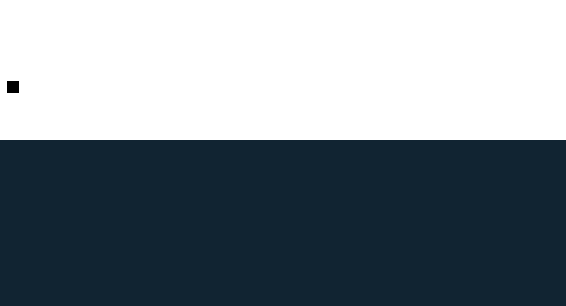

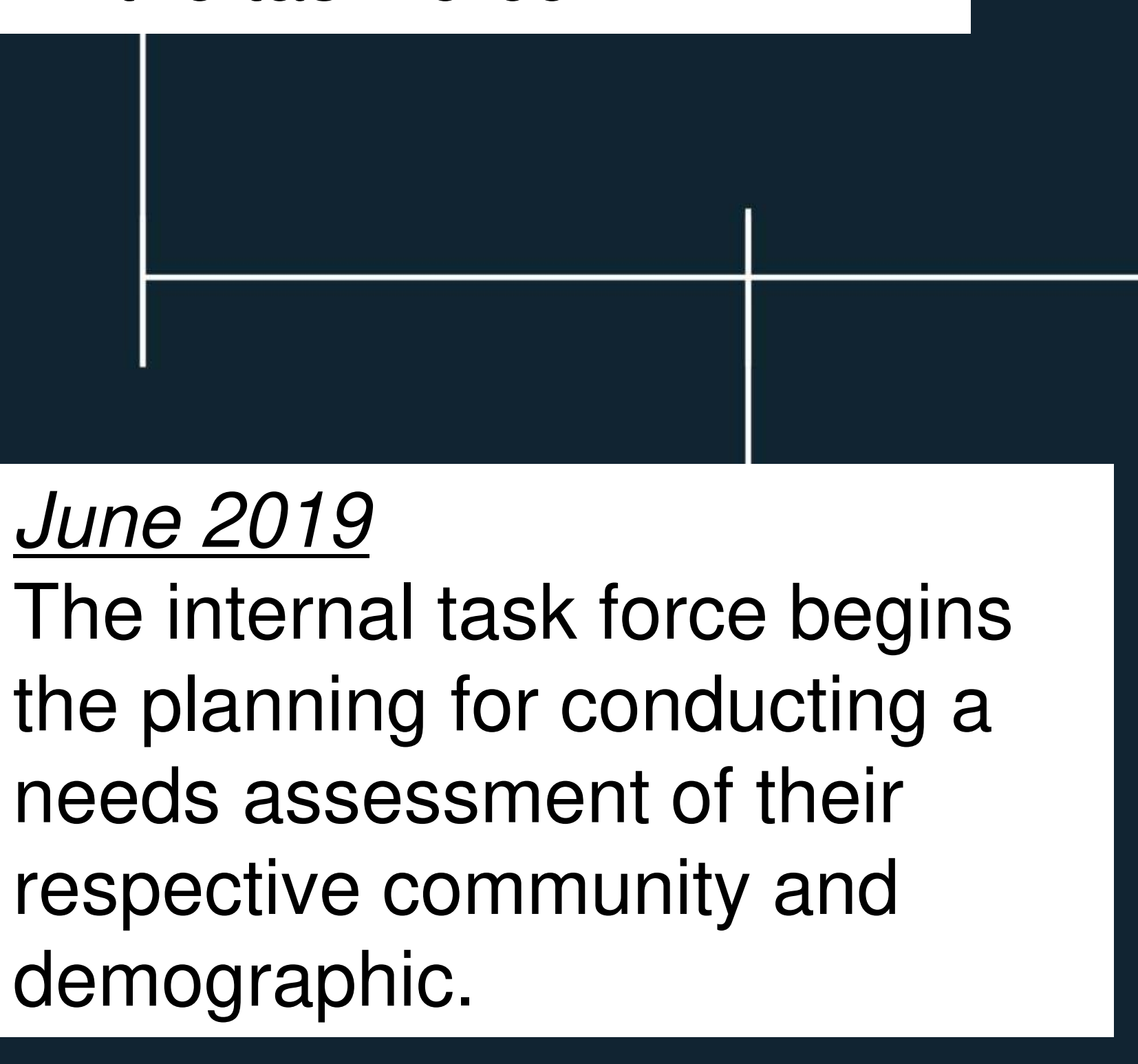
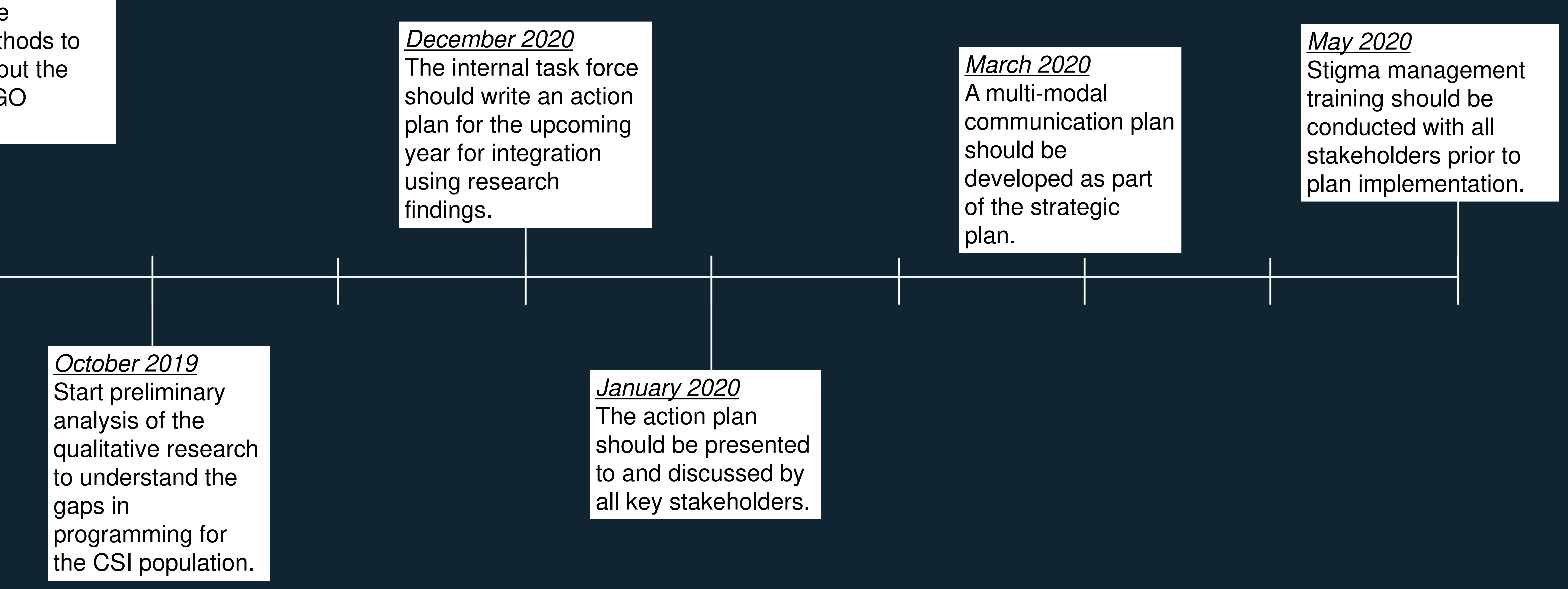